\title{
Sub-lattice polarization states in anti-ferroelectrics and their relaxation process
}

\author{
M.M. Vopson ${ }^{1, *}$, X. Tan ${ }^{2}$, E. Namvar ${ }^{1}$, M. Belusky ${ }^{1}$, S.P. Thompson ${ }^{3}$, V. Kuncser ${ }^{4}$, \\ F. Plazaola ${ }^{5}$, I. Unzueta ${ }^{5}$, C.C. Tang $^{3}$ \\ ${ }^{1}$ School of Mathematics and Physics, University of Portsmouth, Portsmouth PO1 3QL, UK \\ ${ }^{2}$ Department of Materials Science and Engineering, Iowa State University, Ames, Iowa 50011, USA \\ ${ }^{3}$ Diamond Light Source Ltd, Harwell Science and Innovation Campus, Didcot, Oxfordshire OX11 ODE, UK \\ ${ }^{4}$ National Institute for Materials Physics, Bucharest-Magurele, Romania \\ ${ }^{5}$ Department of Electricity and Electronics, UPV/EHU, C/Sarriena s/n, Campus de Leioa 48940 LEIOA, Spain \\ *Corresponding author: melvin.vopson@port.ac.uk, formerly known as Vopsaroiu.
}

\begin{abstract}
We report studies of quasi-remanent polarization states in $\mathrm{Pb}_{0.99} \mathrm{Nb}_{0.02}\left[\left(\mathrm{Zr}_{0.57} \mathrm{Sn}_{0.43}\right)_{0.94} \mathrm{Ti}_{0.06}\right]_{0.98} \mathrm{O}_{3}$ (PNZST) antiferroelectric ceramics and investigation of their relaxation effects using unique in-situ electrically activated time-resolved Synchrotron X-ray powder diffraction (SXPD) and ${ }^{119} \mathrm{Sn}$ Mössbauer Spectroscopy (MS). The SXPD patterns are consistent with a phase transition from quasi-tetragonal perovskite in $0 \mathrm{~V}$ relaxed antiferroelectric state to rhombohedral distortion in ferroelectric state under saturating applied voltages of $\pm 2 \mathrm{kV}$. The observed quasi-remanent polarization relaxation processes are due to the fact that tetragonal to rhombohedral distortion does not occur at the applied voltage required to access the quasi-remanent polarization states, and the tetragonal symmetry restored after the removal of the applied electric field is preserved. Since these quasi-remanent polarization states were seen as possibly suitable for memory applications, the implications of this study are that anti-ferroelectrics are more feasible for multi-state dynamic random access memories (DRAM), while their application to non-volatile memories requires development of more sophisticated "read-out" protocols, possibly involving dc electrical biasing.
\end{abstract}

Keywords: polarization relaxation, anti-polar materials, solid state memories, time resolved synchrotron measurements, Mossbauer spectroscopy

\section{Introduction}

The worldwide demand for digital data storage is increasing exponentially. IBM estimates that 2.5 quintillion of digital data bytes are produced every day on Earth $\left(2.5 \times 10^{18}\right.$ bytes $=$ 2.5 billion Gigabytes). Hence there is a perpetual need to develop new data storage technologies that meet the increasing demand at reduced cost and power consumption. These issues have prompted the acceleration of research into solid-state memories, which are fast replacing traditional magnetic hard disc drives in almost all consumer electronics and portable devices. Recently, studies of anti-ferroelectric materials for memory applications have been the focus of a number of research groups around the World.

Anti-ferroelectric materials in relaxed state consist of two equally and opposing ferroelectric sublattices [1]. Denoting A as the negative ferroelectric sublattice and B as the positive one, an externally applied electric field forces one of the sublattices to undergo reversal into positive or negative polarization, while the other sublattice remains largely unchanged. Hence, the application of a large enough external electric field results in switching of the antiferroelectric from anti-polar to polar ferroelectric. Unlike ferroelectrics that display a single hysteresis loop, anti-ferroelectrics display a double hysteresis PE loop, where each hysteresis loop represents the response of one of the two sublattices. More details on the theory of polarization reversal in anti-ferroelectrics and full explanation of the double hysteresis loop are given in a recent article [2]. However, materials displaying hysteresis are very useful for memory device applications. For example ferroelectric random access memory (FRAM) encodes data in the two remanent polarization states of a ferroelectric [3-5]. Since anti- 
ferroelectrics consist of two ferroelectric sublattices and a double-hysteresis, recently there have been a number of studies proposing possible memory effects in anti-ferroelectrics [6,7]. Vopson et al. have proposed a 4-state non-volatile anti-ferroelectric random access memory device termed AFRAM [8] that could store digital data in quasi-remanent polarization states in each of their two ferroelectric sub-lattices (see A0, A1, B0, B1 in fig.1), while Zhang et al. have reported a tristate non-volatile memory effect in anti-ferroelectrics [9].

However, despite these recent proposals for anti-ferroelectric memory devices, the study of memory effect in anti-ferroelectrics is far from settled. In particular, a polarization relaxation process that can lead to memory self-erasure requires further studies. Understanding the sublattice polarization dynamics in anti-ferroelectrics also helps to define more efficient "write / read" operation protocols for anti-ferroelectric memories. In to clarify these issues, here we examine the anti-ferroelectric quasi-remanent polarization states in great detail using polarization retention measurements and in-situ time-resolved Synchrotron experiments, in addition to probing possible hyperfine interactions via $\mathrm{Sn}^{119}$ Mössbauer Spectroscopy.

\section{Experiments}

\subsection{Quasi-remanent polarization retention studies}

Experiments were carried out on $\mathrm{Pb}_{0.99} \mathrm{Nb}_{0.02}\left[\left(\mathrm{Zr}_{0.57} \mathrm{Sn}_{0.43}\right)_{0.94} \mathrm{Ti}_{0.06}\right]_{0.98} \mathrm{O}_{3}$ anti-ferroelectric ceramic (sample code PNZST). Full details of the sample preparation process can be found in [10]. The sample is a $10 \mathrm{~mm}$ diameter, $616 \mu \mathrm{m}$ thick ceramic disk with metallic electrodes applied on each side of the ceramic.

Polarization hysteresis loops and quasiremanent memory retention experiments were taken using an AiXact 2000E PiezoTest Analyser. All measurements were performed at room temperature. Hysteresis loops were acquired using a triangular field waveform of $2 \mathrm{kV}$ amplitude and $0.1 \mathrm{~Hz}$ frequency, with a pre-polarization pulse applied first. Figure 1 represents a room temperature hysteresis loop for PNZST anti-ferroelectric ceramic. As indicated in fig. 1, there are four possible quasiremanent memory states, two for each ferroelectric sublattice. The data "write" and "read" operations are accomplished by the application of pairs of saturation

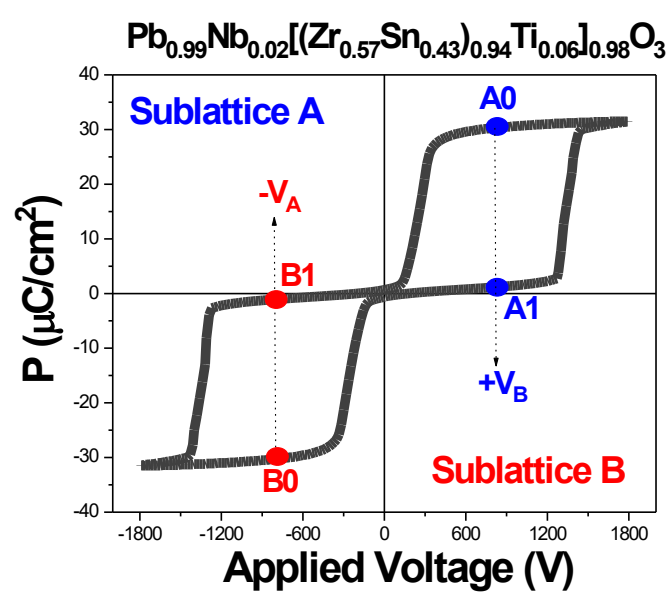

Figure 1. Experimental PE loop of PNZST ceramic showing the quasi-memory states and the activation voltages. voltages $\pm V_{s}$ for the "write" operation, and activation voltages $-V_{A}$ or $+V_{B}$ for the "read" operation, as shown in fig. 1. The four quasiremanent memory states can be extracted using four possible permutations of the applied pulses: $+\mathrm{V}_{\mathrm{s}} /+\mathrm{V}_{\mathrm{B}}$ for $\mathrm{A} 0,+\mathrm{V}_{\mathrm{s}} /-\mathrm{V}_{\mathrm{A}}$ for $\mathrm{B} 1,-\mathrm{V}_{\mathrm{s}} /-\mathrm{V}_{\mathrm{A}}$ for $\mathrm{B} 0$ and $-\mathrm{V}_{\mathrm{s}} /+\mathrm{V}_{\mathrm{B}}$ for $\mathrm{A} 1$, respectively. From hysteresis measurements we determined the saturation voltage $\mathrm{V}_{\mathrm{s}}= \pm 1.8 \mathrm{kV}$ and the activation voltages $\mathrm{V}_{\mathrm{A}, \mathrm{B}}= \pm 760 \mathrm{~V}$. The anti-ferroelectric quasi-remanent memory retention experiments were conducted by deploying a measurement test sequence that involved the following steps: a) apply a "write" saturating pulse, $\mathrm{V}_{\mathrm{s}}= \pm 1.8 \mathrm{kV}$; b) go to zero field; c) wait for a time, "delay-to-read"; d) apply the activation voltage "read" pulse $\mathrm{V}_{\mathrm{A}, \mathrm{B}}= \pm 760 \mathrm{~V}$ and measure the quasi-remanent polarization. Both "write" and "read" electrical pulses were trapezoidal with three time-equal branches. The total duration of the "write" pulse was $3 \mathrm{~s}$ and 
the duration of the "read" pulse was $450 \mathrm{~ms}$, with measurements of the quasi-remanent polarizations taken at $\mathrm{t}=300 \mathrm{~ms}$. The "delay-to-read" time was variable having the values $0.1,1,10$ and 100 seconds, respectively, while longer waiting times were inaccessible to our experimental apparatus. For each of the "wait" times, we successfully extracted the four quasi-remanent polarization states and a very distinguishable response can be observed. First, the quasi-remanent polarization values corresponding to A0, A1, B0, B1 were extracted from the PE hysteresis loop. These values are denoted as "delay-to-read" time $=0$ seconds in Table 1. In the same table we listed the quasi-remanent polarization values corresponding to the four memory states extracted at four different waiting times.

Table 1. Quasi-remanent polarization memory states of PNZST anti-ferroelectric measured on the double hysteresis loop (marked as 0 seconds) and their values at four incremental waiting times.

Memory States

Delay-to-Read time (s)

\begin{tabular}{cccc}
\multicolumn{4}{c}{ Memory States } \\
Quasi-Remanent & Polarization & $\left(\mu \mathrm{C} / \mathrm{cm}^{2}\right)$ \\
\hline A0 & A1 & B0 & B1 \\
30 & 1.37 & -30 & -1.35 \\
3.92 & 0.02 & -3.26 & -0.024 \\
1.73 & 0.014 & -2.16 & -0.015 \\
0.16 & 0.015 & -0.13 & -0.014 \\
0.06 & 0.013 & -0.046 & -0.012 \\
\hline
\end{tabular}

The values shown in Table 1 indicate that, although the four distinguishable states can be extracted after a given "wait" time, there is a clear and significant decay in these values as a function of the waiting time. The significant reduction in quasi-remanent polarization values from 0 to 0.1 seconds corre3sponding to the first delay-to-read time, represents the reversible phase transformation from rhombohedral to tetragonal. The further polarization reductions observed for incremental delay-to-read times are indicative of a relaxation process. Figures 2 $\mathrm{a}, \mathrm{b}$ show a graphical representation of the polarization decay as a function of the waiting time.

a)

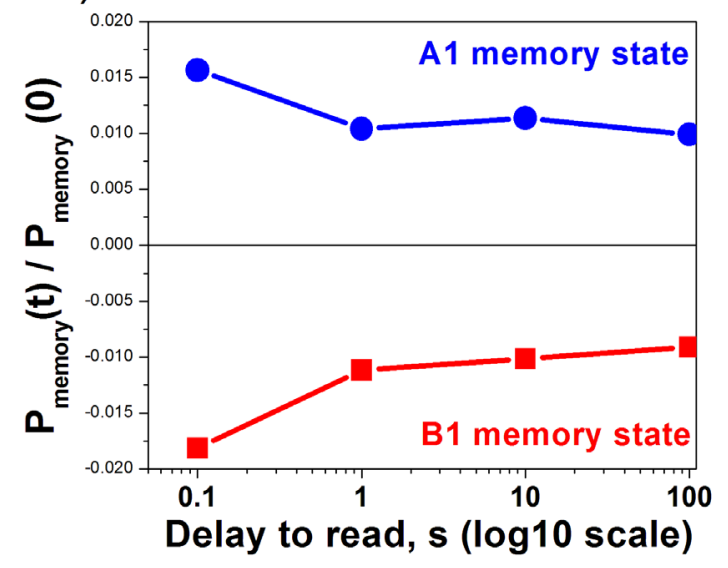

b)

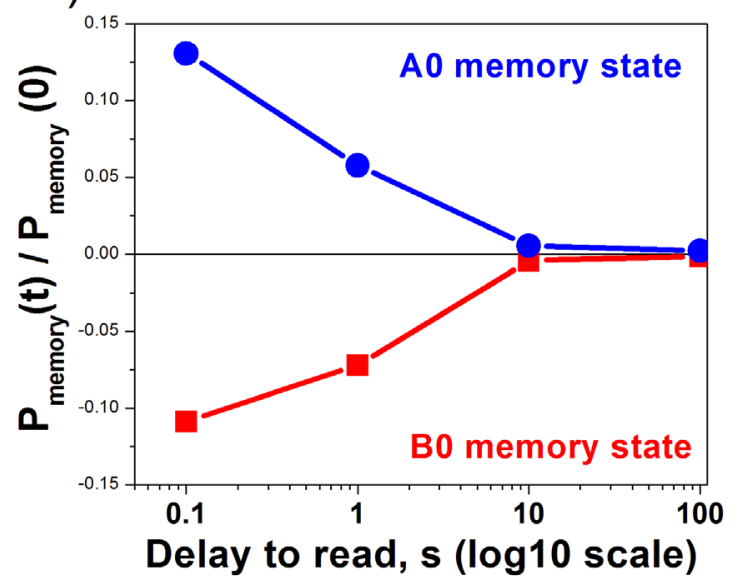

Figure 2. a) Values of A1, B1 and b) A0, B0 quasi-polarization states at incremental "delay-to-read" values. For better representation, data is plotted in logarithmic scales and polarization values are normalized to the maximum quasi-remanent polarization value, measured on the PE loops.

This relaxation process weakens the retention capacity of the anti-ferroelectric memory and can lead to memory self-erasure, or the loss of non-volatility. 


\subsection{In-situ time-resolved Synchrotron X-ray powder diffraction (SXPD)}

The nature of this relaxation process is unclear and it is the subject of investigation in this article. One possible explanation could be related to fine structural changes and, in order to understand this effect at a fundamental level, it is important to probe these atomic-scale structural changes by measuring the diffraction pattern under an applied dc electrical field using X-ray or other diffraction methods, as already reported for ferroelectric materials [1115]. Here we performed in-situ Synchrotron X-ray powder diffraction (SXPD) on antiferroelectric ceramics under applied voltages / electric fields using the high-resolution SXPD beamline I-11 [16] at Diamond Light Source in the UK. In order to understand the dynamics of the observed relaxation mechanism, time-resolved diffraction patterns have also been collected. The beamline has high brightness X-ray beam with an optimum energy of $15 \mathrm{keV}$. The beam size used for the experiment was $100 \mu \mathrm{m}$ (vertical) $\times 2.5 \mathrm{~mm}$ and the wavelength $(\lambda=0.82466(2) \AA$ ) $)$ was calibrated from a pattern of high quality $\mathrm{Si}$ powder standard (SRM640c). With the beam incident at sample surface, diffraction patterns were collected with a curved Position Sensitive Detector (PSD) which has $90^{\circ}$ coverage in $2 \theta$. The PSD allows acquisition of high-resolution SXPD data in as little as one second per pattern. More technical details of the beamline and the detector performance can be found in reference [17]. In order to perform in-situ electrically activated diffraction measurements on antiferroelectrics, a special sample holder consisting of a printed circuit board (PCB) stage with suitable electrical connections was used. The anti-ferroelectric sample was mounted with conductive silver loaded epoxy onto the PCB stage. The PCB is mounted on a motorized XYZ stage capable of several millimeters travel for sample alignment. The in-situ sample stage was previously designed for users of beamline I11 facility primarily for testing ferroelectric materials under electric field excitation [18]. To polarize the sample electrically an Agilent 33220A function generator was used to output 0 to $\pm 10 \mathrm{~V}$ de voltage that was used as input to a TREK $610 \mathrm{E}$ high voltage amplifier. The final output of $\pm 1 \mathrm{kV}$ or \pm 10 $\mathrm{kV}$ from the amplifier was selected to activate the sample. The measurements have been performed at room temperature (RT). Figure 3 shows the diffraction pattern of PNZST ceramic at low angles collected in relaxed state under zero applied voltage with the sample surface set at $10^{\circ}$. The data indicates a pseudotetragonal perovskite

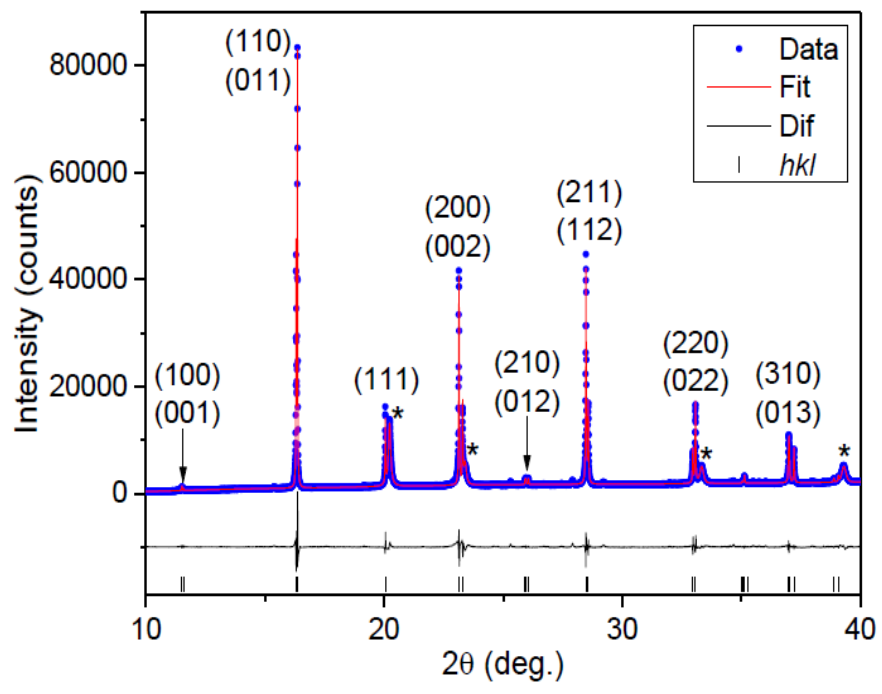

Figure 3. SXPD pattern of PNZST anti-ferroelectric ceramic measured in zero applied electric field/voltage $(\lambda=0.82466 \AA)$. The broad peaks ${ }^{*}$ ) were produced by the thin Ag coating for electrodes.

symmetry in the virgin state

with lattice parameters $\mathrm{a}=\mathrm{b}=4.11111(2) \AA, \mathrm{c}=4.08410(2) \AA$, and a unit cell volume of $\mathrm{V}_{0}$ $=69.0262 \AA^{3}$ consistent with previous reports $[10,19,20]$. These were obtained by a good profile fit (red curve) to the data (blue points) as indicated by the difference (bottom black trace). Patterns were then collected with the in-situ E field / voltage applied. Although the PE 
hysteresis loops indicate a $1.8 \mathrm{kV}$ saturation voltage, during synchrotron measurements we used $\pm 2 \mathrm{kV}$ in-situ saturation voltage. Upon the application of the saturation voltage, the unit cell volume undergoes an expansion to $\mathrm{V}^{+}=69.0972 \AA^{3}$ under $+2 \mathrm{kV}$ field, and $\mathrm{V}^{-}=69.1580$ $\AA^{3}$ under $-2 \mathrm{kV}$, resulting in volume strains $\varepsilon_{+,-}=\left(\mathrm{V}^{+,-}-\mathrm{V}_{0}\right) / \mathrm{V}_{0}$ of 0.001 and 0.002 , respectively. Figure 4 shows a selection of diffraction peaks for the sample under zero voltage and the same peaks acquired under positive and negative saturating voltages. Peak (211) shows a double splitting when the sample is in relaxed state and a triple splitting when the sample is saturated in a positive or negative electric field / voltage (Fig. 4.a). Family peaks (110) and (220) display a double splitting in relaxed state and maintain a double splitting when polar phase is induced by the saturating electric field (Fig. 4.b,d). Finally the (111) peak shows a single line in relaxed state and this becomes a doublet in polar phase under applied $\pm 2 \mathrm{kV}$ (Fig. 4.c). These peak splitting patterns are fully consistent with the tetragonal to rhombohedral distortion [10] induced by the application of the E field / voltage, which is essentially equivalent with a transition from anti-ferroelectric to ferroelectric phase. When the applied voltage is reduced to zero, the anti-ferroelectric phase with tetragonal distortion is always fully restored.
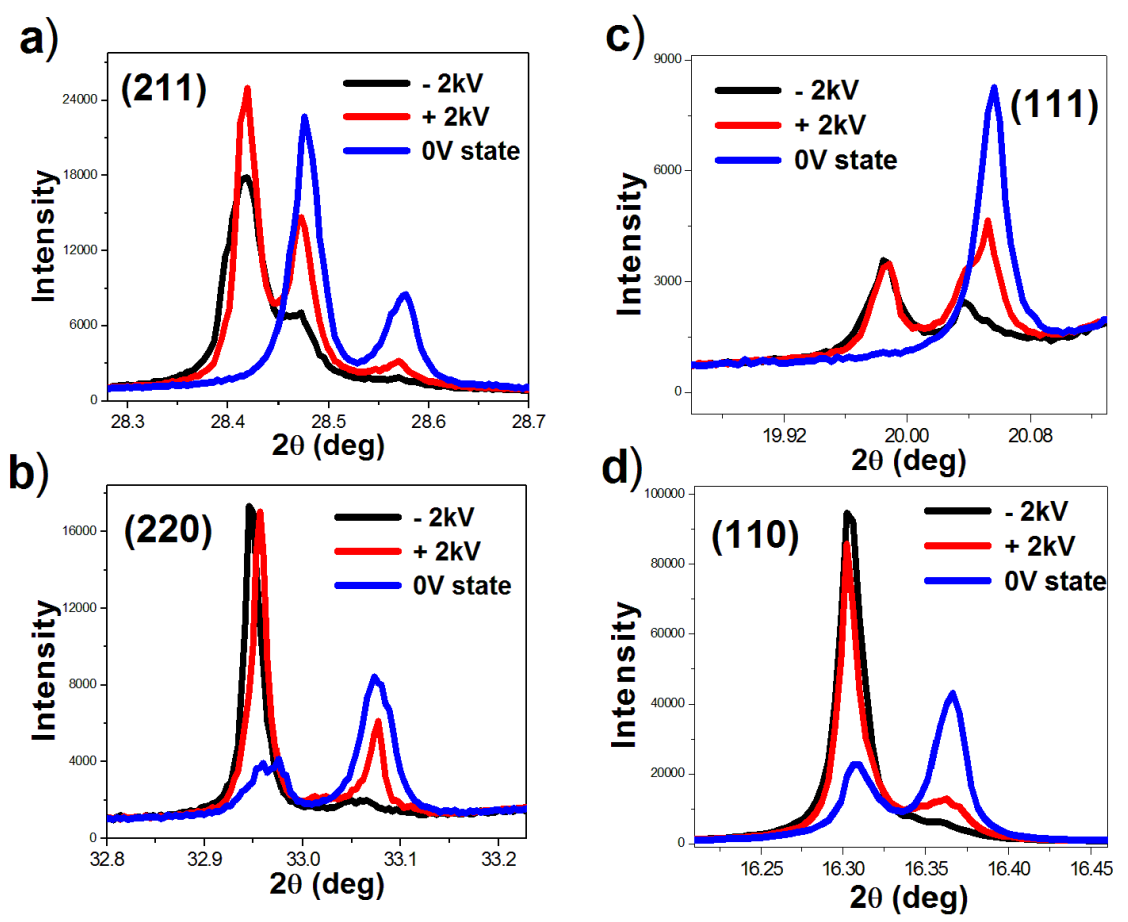

Figure 4. SXPD data of PNZST anti-ferroelectric on selected peaks probed under relaxed state $(0$ applied voltage), and saturating positive $(+2 \mathrm{kV})$ and negative $(-2 \mathrm{kV})$ voltages. a) Peak $(211)$; b) Peak (220); c) Peak (111); d) Peak (110).

The PSD was also used for time-resolved study of the polarization relaxation process. The dynamics of the memory states of the anti-ferroelectric were studied by taking in-situ diffraction readings at the activation voltages, at different time intervals after the application of the "write" voltage pulse and subsequent switching back to $0 \mathrm{~V}$ after the "write" operation. The experiment involved the exact protocol used to extract the memory states from electrical / polarization measurements: a) Saturate the sample by applying $V_{s}= \pm 2 k V$ voltage; $b$ ) Go to zero field, $\mathrm{V}=0 \mathrm{~V}$; $\mathrm{c}$ ) Wait in relaxed zero field state for a time $\mathrm{t}=10$ and 100 seconds; $\mathrm{d}$ ) Access the quasi-remanent memory states by applying the activation voltage, $\mathrm{V}_{\mathrm{A}, \mathrm{B}}= \pm 760 \mathrm{~V}$; e) Acquire X-ray data while the activation voltage is ON. The 10 and 100 seconds "waiting" 
times were selected to match the "delay-to- read" times deployed during the quasi-remanent polarization retention measurements (see Table 1), except that the very short 0.1 and 1 second waiting times were not accessible during the in-situ time resolved synchrotron studies. The four quasi-remanent memory states can be extracted using four possible permutations of the applied pulses: $+V_{s} /+V_{B}$ for $A 0,+V_{s} /-V_{A}$ for $B 1,-V_{s} /-V_{A}$ for $B 0$ and $-V_{s} /+V_{B}$ for $A 1$, respectively. By choosing the correct sequence of applied "write" voltage and activation "read" voltage, the dynamics of the relaxation process of the quasi-remanent memory states can be observed by monitoring the X-ray diffraction as a function of the "waiting" time. At a closer inspection of the time resolved diffraction patters we identified only the (211) and (220) peaks to display visible relaxation response that correlates to the sequence of applied fields to activate the four memory states. The (211) peak corresponds to $A 1\left(-V_{s} /+V_{B}\right)$ or $B 0$ $\left(-\mathrm{V}_{\mathrm{S}} /-\mathrm{V}_{\mathrm{A}}\right)$ memory states, which are linked by the fact that both require the application of $\mathrm{V}_{\mathrm{S}}$ $=-2 \mathrm{kV}$ "write" voltage, while the relaxations of $\mathrm{B} 1\left(+\mathrm{V}_{\mathrm{s}} /-\mathrm{V}_{\mathrm{A}}\right)$ and $\mathrm{A} 0\left(+\mathrm{V}_{\mathrm{s}} /+\mathrm{V}_{\mathrm{B}}\right)$ memory states are monitored via (220) peak, with both states being activated by the $\mathrm{V}_{\mathrm{s}}=+2 \mathrm{kV}$ "write" voltage. Figure 5 shows the diffraction peaks corresponding to each memory state acquired at 10 and 100 seconds "delay-to-read" (or waiting) times. These are plotted together with the diffraction peaks in relaxed zero voltage state of the anti-ferroelectric. The quasiremanent memory relaxation is monitored as peak intensity change, with a clear trend identified showing that the longer is the waiting time between "write" and "read" operations, the closer the observed peak intensity is to the peak corresponding to zero voltage relaxed state. In the case of memory states A1 and B0 activated by a negative "write" voltage, this is translated into the (211) peak intensity decrease for longer waiting times, while for B1 and A0 memory states activated by positive "write" voltage it results in the increase of the (220) peak intensity for longer waiting times.
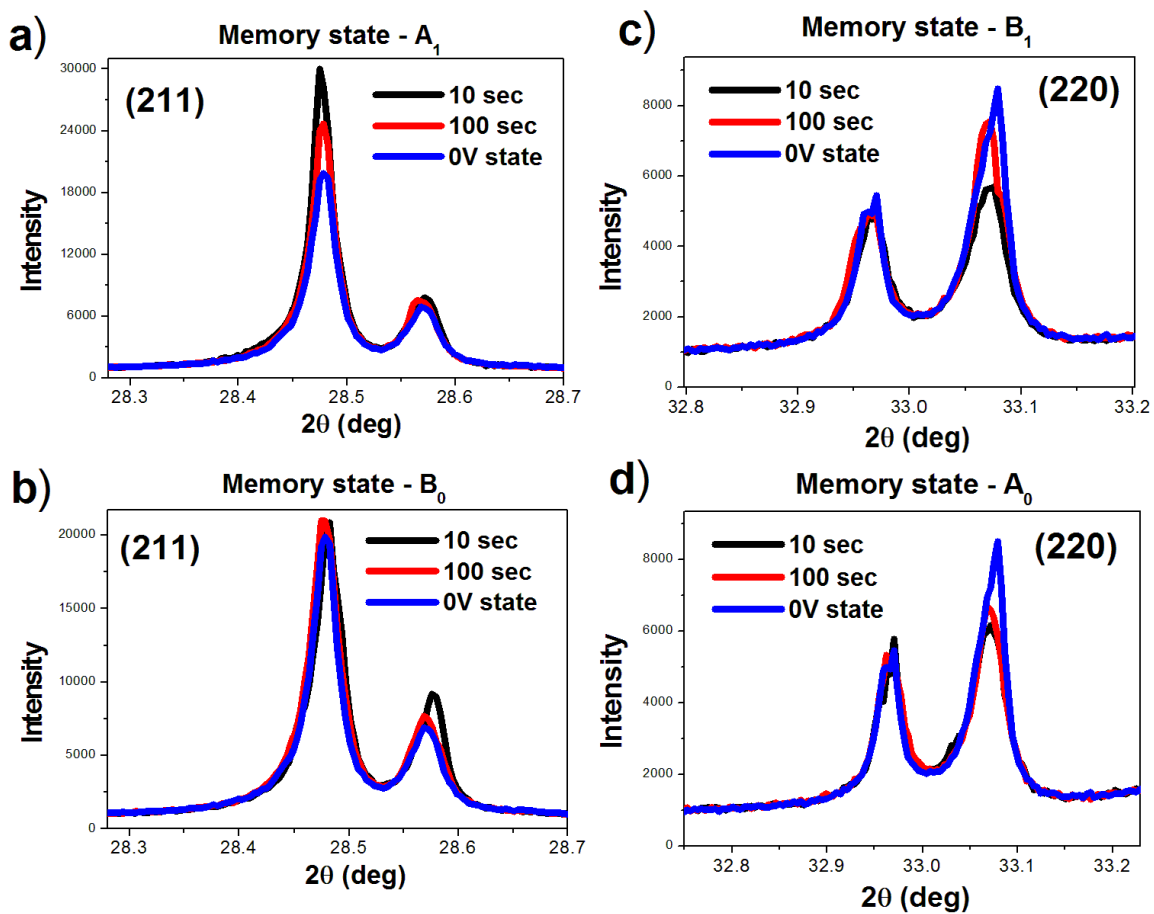

Figure 5. Time-resolved SXPD measurements probing the anti-ferroelectric sample at the exact applied field sequences corresponding to the four quasi-remanent memory states, at two waiting times (10 and 100 seconds) and relaxed zero field state. Relaxations process clearly visible for selected peaks corresponding to each memory state.

To understand these results we recall that a polycrystalline ceramic sample should contain large number of crystallites. The X-ray diffraction provides information from the coherent 
diffraction domains, which are the largest regions in three-dimensional space that satisfy the periodic translation of the crystal unit-cell [21-23].

Grains in anti-ferroelectrics contain mostly anti-ferroelectric domains, and possibly some ferroelectric domains. These domains may show diverse orientations of the spontaneous polarizations, so in an anti-ferroelectric or ferroelectric, the coherent diffraction domains coincide with the polar / anti-polar domains. Applying a large enough voltage to a polycrystalline anti-ferroelectric is a process that induces a phase transition from anti-polar to polar ferroelectric, increasing the preferential orientation since it increases the volume fraction of ferroelectric domains with their polarizations as close as possible to the applied electric field direction, and reducing the volume of the less favourably aligned domains. Therefore, we expect a growth in the peaks intensity of the X-ray diffraction pattern corresponding to inter-planar distances parallel with the applied electric field, and a decrease of peaks intensity corresponding to inter-planar distances perpendicular to the applied electric field direction. This is exactly what it has been observed in our time resolved in-situ synchrotron measurements. The quasi-tetragonal symmetry, restored from rhombohedral distortion during the "write" process, appears to be maintained when the anti-ferroelectric sample is subjected to the activation voltages $V_{A}$ and $V_{B}$ where the memory states are accessed. This is evidenced by the lack of peak splitting indicative of rhombohedral distortion at the applied "read" voltage (see figure 5). The time relaxation process indicates peak intensity increasing under applied voltage for (211), which corresponds to inter-planar distance almost parallel to the $\mathrm{E}$ field direction, and peak intensity decreasing at the activation voltage for (220), which has inter-planar distance perpendicular to the applied electric field direction.

\subsection{Mössbauer spectroscopy}

$\mathrm{Sn}^{119}$ Mössbauer Spectroscopy experiments have been performed on our Sn containing antiferroelectric complex ceramic in order to figure out possible electron delocalization phenomena and other revealing hyperfine interactions at $\mathrm{Sn}$ sites. Room temperature Mössbauer spectra were obtained at maximum speed of $7 \mathrm{~mm} / \mathrm{s}$ using a $\mathrm{Ba}^{119}$ $\mathrm{SnO}_{3}$ source in transmission setup equipped with a triangle-wave generator. In order to reduce the background noise of spurious X-rays emissions of 25 $\operatorname{keV}(\mathrm{SnK} \alpha)$ and $29 \mathrm{keV}(\mathrm{SnK} \beta)$, which take place along with the Mössbauer gamma ray emission (23.8 $\mathrm{KeV}$ ), two Pd foils $25 \mu \mathrm{m}$ thick have been placed between the source and the sample. The spectrometer was calibrated using $\mathrm{SnO}_{2}$ [24,25]. The NORMOS program developed by Brand has been used to fit the spectra [26]. Figure 6 shows the room temperature Mössbauer spectrum of the PNZST anti-ferroelectric ceramic in powder form, together with the typical absorption spectrum of the $\mathrm{SnO}_{2}$ calibration absorber. The spectrum indicates the lack of quadrupole splitting,

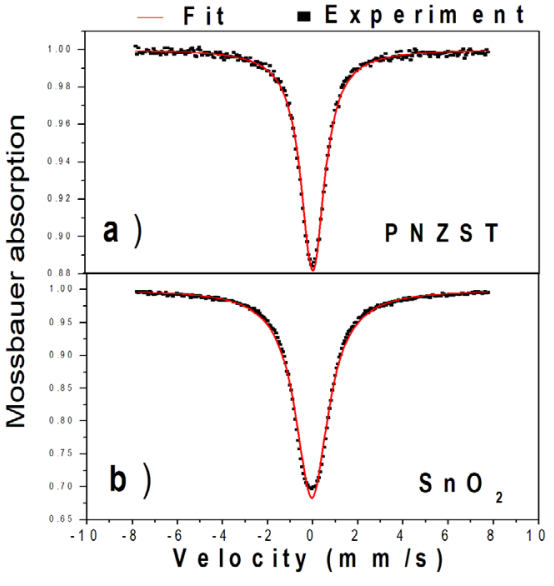

Figure 6. Mössbauer spectra of powder samples of a) PNZST; b) $\mathrm{SnO}_{2}$ calibration absorber. or magnetic hyperfine field present at the Sn sites, while the isomer shift is very close to zero. Relative to the $\mathrm{SnO}_{2}$ absorber, the isomer shift value was determined to be $0.064 \mathrm{~mm} / \mathrm{s}$. Since the $\mathrm{Ti}$ dynamics drives the polarization behaviour in these compounds, measurements not shown here on other samples of identical structure, but with slightly different $\mathrm{Ti}$ concentration resulted in similar results. These values indicate an oxidation state of IV for the 
Sn ions, with the spectrum showing no trace for any other different electron configuration of $\mathrm{Sn}$. The rather narrow spectral line (i.e. $1.28 \mathrm{~mm} / \mathrm{s}$ at half-width), also suggests a lack of sensible crystal field distortion at the Sn site, implying that their contribution to the spontaneous polarization of the two sublattices is negligible.

\section{Conclusions}

We conclude that the anti-ferroelectric solids are promising candidates for the development of digital memories. However, the application of anti-ferroelectrics to non-volatile memories is questionable because of the observed relaxation processes. The quasi-remanent polarization memory states, although distinguishable, suffer a significant time degradation measured over the 0 - 100 seconds range. This suggests the possibility of using antiferroelectrics at least for multi-state dynamic random access memories (DRAM). Our timeresolved synchrotron studies indicate that the observed relaxation process in the antiferroelectrics is related to a structural relaxation due to the fact that the activation voltages required for the "read-out" process are not sufficiently large to induce the rhombohedral distortion, so the quasi-remanent polarization values are far lower than those observed on PE hysteresis loops. These studies will hopefully stimulate further research into anti-ferroelectric memories including development of better memory "read-out" protocols to minimize the observed memory relaxation process.

\section{Acknowledgements}

MV would like to acknowledge the DLS and EPSRC financial support received for this research through beamline EE18495 and grant EP/R028656/1, respectively. The authors also acknowledge the technical support during the synchrotron measurements received from Stuart Gurney and Jonathan Potter.

\section{References}

[1] C. Kittel, Phys. Rev. 82: 729-732 (1951).

[2]. M. Vopson, X. Tan, Physical Review B 96 (1), 014104 (2017).

[3] H. Ishiwara, Ferroelectric Random Access Memories, J. Nanosci. Nanotechnol. 12, 7619-7627, (2012).

[4] R. Guo, L. You, y. Zhou, Z.S. Lim, X. Zou, L. Chen, R. Ramesh, J. Wang, Nature Communications 4:1990 (2013).

[5] Y. Kato, Y. Kaneko, H. Tanaka, K. Kaibara, S. Koyama, K. Isogai, T. Yamada, Y. Shimada, Overview and future challenge of ferroelectric random access memory technologies. Jpn. J. Appl. Phys. 46, 2157 (2007).

[6] M. Pešic, M. Hoffmann, C. Richter, T. Mikolajick, and U. Schroeder, Adv. Funct. Mater. 26, 7486 (2016).

[7]. M. Vopson, G. Caruntu, X. Tan, Scripta Materialia vol. 128, 61-64 (2017).

[8] M. Vopson, X. Tan, Electron Device Letters (2016).

[9] H. Zhang, J. Zhou, J. Shen, T. Wang, D. Xie, W. Chen, Appl. Phys. Lett. 113, 152902 (2018)

[10] X. Tan, S.E. Young, Y.H. Seo, J.Y. Zhang, W. Hong, K.G. Webber, Acta Materialia 62, 114-121 (2014)

[11] R. C. Rogan, E. Üstündag, B. Clausen, and M. R. Daymond, J. Appl. Phys., vol. 93, no. 7, p. 4104, 2003.

[12] Jones, J. L., Hoffman, M., Daniels, J. E. \& Studer, A. J. (2006). Appl. Phys. Lett. 89, 092901.

[13] Daniels, J. E., Finlayson, T. R., Davis, M., Damjanovic, D., Studer, J., Hoffman, M. \& Jones, J. L. (2007). J. Appl. Phys. 101, 104108.

[14] Pramanick, A., Prewitt, A. D., Cottrell, M. A., Lee, W., Studer, A. J., An, K., Hubbard, C. R. \& Jones, J. L. (2010). Appl. Phys. A, 99, 557-564.

[15] Moriyoshi, C., Hiramoto, S., Ohkubo, H., Kuroiwa, Y., Osawa, H., Sugimoto, K., Kimura, S., Takata, M., Kitanaka, Y., Noguchi, Y. \& Miyayama, M. (2011). Jpn. J. Appl. Phys. 50, 09NE05. 
[16] Thompson, S., Parker, J., Potter, J., Hill, T., Birt, A., Cobb, T., Yuan, F. \& Tanng, C. (2009). Rev. Sci. Instrum. 80 (7), 075107.

[17] Thompson, S., Parker, J., Marchal, J., Potter, J., Birt, A., Yuan, F., Fearn, R., Lennie, A., Street, S. \& Tang, C. J. Synchrot. Radiat. 18 (Part 4), 637-648 (2011).

[18] S. Ryding, R. Cernik, J. Wooldridge, T.L. Burnett, M. Stewart, C. Vecchini, M.G. Cain, A. Lennie, F. Yuan, C. Tang, P. Thompson, Powder Diffraction, Vol. 28, Issue S2, pp. S220-S227 (2013).

[19] H. Guo, X. Tan, Phys. Rev. B 91, 144104 (2015)

[20] W. Qu, X. Tan, Thin Solid Fils 496, 383-388 (2006)

[21] Cullity, B.; Stock, S. Elements of X-ray Diffraction, 3rd ed.; Prentice-Hall: Upper Saddle River, NJ, USA, 2001.

[22] Guinebretière, R. X-Ray Diffraction by Polycrystalline Materials; ISTE Ltd.: London, UK, 2013.

[23] J. E. Daniels, J. L. Jones, and T. R. Finlayson, J. Phys. D. Appl. Phys. 39, 5294 (2006).

[24] R. H. Herber, Phys. Rev. B 27, 4013 (1983)

[25] A. Svane, N. E. Christensen, C. O. Rodriguez and M. Methfessel, Phys. Rev. B 55, 18 (1997).

[26] R.A. Brand, Nuclear Instruments and Methods B, 28, Issue 3 (1987) 398 - 416. 